\title{
LEXICAL ACCESS IN SPEECH PRODUCTION
}

\section{INTRODUCTION}

Lexical access in speech production proceeds at a rate of, on the average, two to three words per second. At this rate words are selected from a production lexicon which contains thousands, and probably tens of thousands, of words. These words are not only selected, but also phonologically encoded. This happens at a rate of about 15 speech sounds per second. The problem to be addressed in this chapter is how these high-rate and fairly accurate processes of lexical selection and phonological encoding are organized.

Figure 1 outlines a possible architecture for the organization of these processes of lexical access. There is a so-called "formulator" receiving as input the (lexical) concept-to-be-expressed (usually as part of a larger

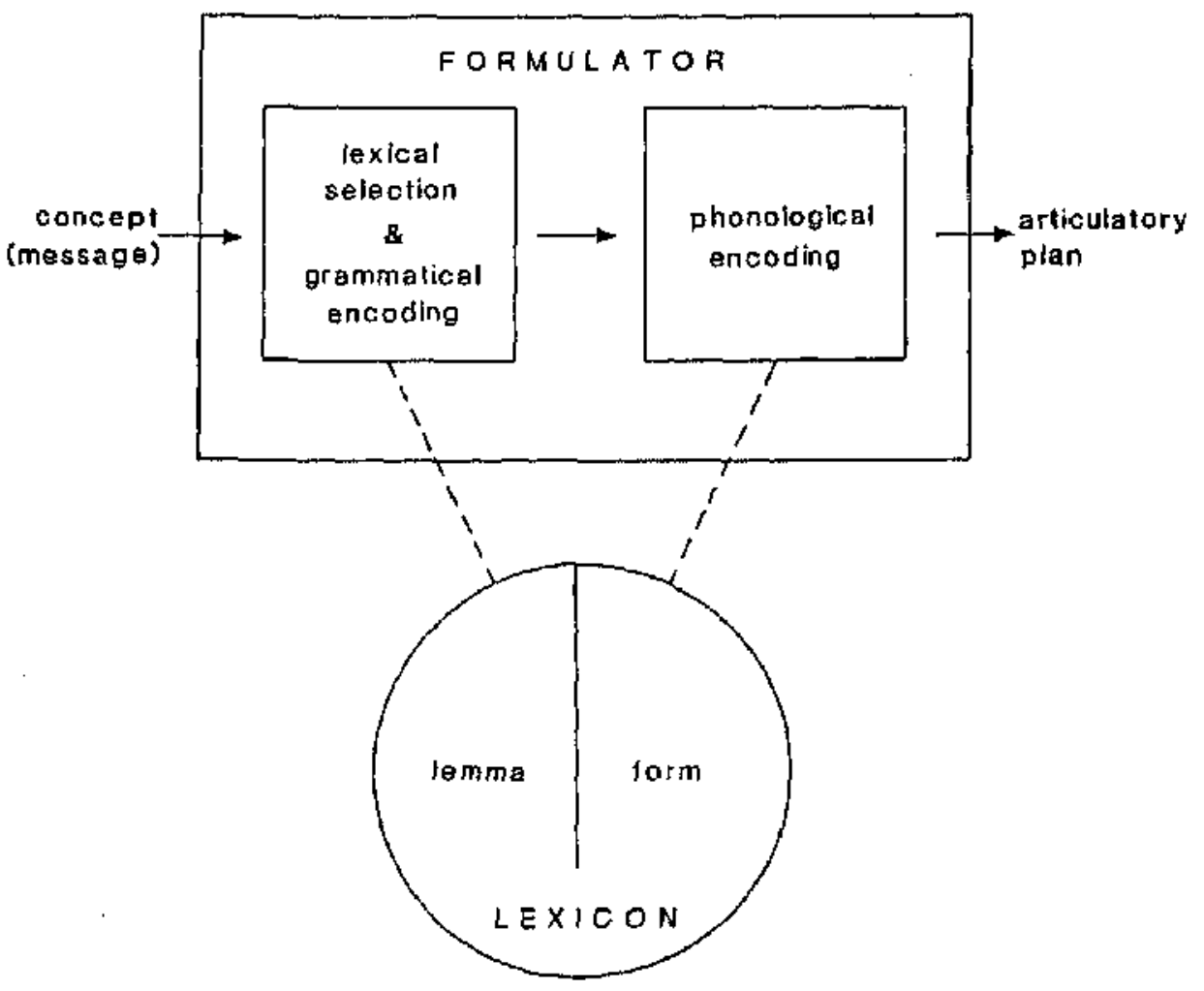

Fig. 1. Outline of a possible architecture for lexical access.

Eric Reuland and Werner Abraham (eds), Knowledge and Language, Volume I, From Orwell's Problem to Plato's Problem: 241-251.

1993 by Kluwer Academic Publishers. Printed in the Netherlands. 
conceptualization) and producing as output an articulatory plan for the item (usually as part of a plan for a larger utterance). The formulator contains two component processors. The first one takes care of selecting the appropriate lexical item from the mental lexicon and of integrating it in the developing syntactic structure (grammatical encoding). The second one generates an articulatory program for the selected lexical item on the basis of its stored phonological code and the developing phonological context of the utterance as a whole.

Each of these two component processes may occasionally derail. If lexical selection goes awry, then errors such as these may occur:

Errors of lexical selection and grammatical encoding.

- Don't burn your toes (intended: fingers).

- Examine the horse of the eyes (intended: the eyes of the horse).

Failing phonological encoding leads to a very different kind of error:

Errors of phonological encoding.

- Fart very hide (intended: fight very hard)

- Face spood (intended: space food)

There are, in particular, three major issues to be asked with respect to the architecture in Figure 1. Two of these will be addressed in the present paper. The first one is the organization of lexical selection. What kind of architecture can mediate between conceptual structure (intentions, concepts to be expressed) and the knowledge base which is called the "mental lexicon", the speaker's store of information about the words of his or her language? The second issue is the structure of phonological encoding. Once an item has been retrieved from the mental lexicon, how is an articulatory program constructed out of the phonological information that is stored with the item? The third issue concerns the temporal alignment of lexical selection and phonological encoding. Are these two processes strictly successive in time, or can an item's phonological encoding begin before it is definitely selected?

Psycholinguistic research has largely concentrated on the second of these three issues, phonological encoding. I will, however, ignore it here (see Levelt (1989) for an extensive review of phonological encoding), and concentrate on lexical selection and on the time course issue.

\section{LEXICAL SELECTION}

How does the speaker select the appropriate word for the expression of the concept at hand? There are some rather heterogeneous proposals about the organization of this process (such as Morton's logogen theory, Goldman's discrimination net theory, Miller and Johnson-Laird's decision 
table theory, and the various connectionist suggestions, all reviewed in Levelt (1989)). What they have in common, however, is that they fail to solve what I have called the hyperonym problem. It can be formulated as follows:

The hyperonym problem. When item B's meaning entails item A's meaning, $\mathrm{A}$ is a hypernym of $\mathrm{B}$. If B's semantic conditions are met, then A's are necessarily also satisfied. Hence, if $B$ is the appropriate lexical item, $A$ will (also) be retrieved.

So, for instance, animal is a hyperonym of bear. If the speaker intends to express the concept BEAR, then the semantic conditions for bear are satisfied, but - by implication - also those for animal. Hence animal will be retrieved (as well). All presently existing published theories fall in this hyperonym trap.

In order to solve this problem I have proposed that theories should implement two principles, the specificity principle and the core principle:

The specificity principle. Of all lexical items whose semantic conditions are satisfied by the concept to be expressed, the most specific one (the one with the largest potential of entailment) is to be selected.

The core principle. Each lexical item has a unique (set of) semantic core condition(s). An item is only retrieved if its core is satisfied by the concept to be expressed.

The specificity principle prevents that a target's hyperonym is selected (e.g. animal instead of dog). The core principle prevents that a target's subordinate is selected (e.g. dog instead of animal).

Ardie Roelofs, Manfred Bierwisch and I are presently developing an implementation of these principles in a logical network (see Figure 2).

Figure 2 shows a level of conceptual components or predicates, a level of semantic decision making, and a level of lexical items (more precisely: lemmas, i.e., lexical items without phonological specification). At the level of semantic decision making semantic functions are computed, given the input from the conceptual components. If a function evaluates to "true", activation is propagated to the lexical item on the next Ievel. Such a function has, among others, two crucial properties. The first one is that its evaluation to true is only possible if its core component(s) is (are) satisfied. Second, the function cannot evaluate to true if it receives input from a hyperonym's core component. This "logical structure" of the network is complemented by an "activation structure" which handles the flow of information over time from the conceptual level to the lexical level. In order for an item to be selected, it is not only necessary that its test 


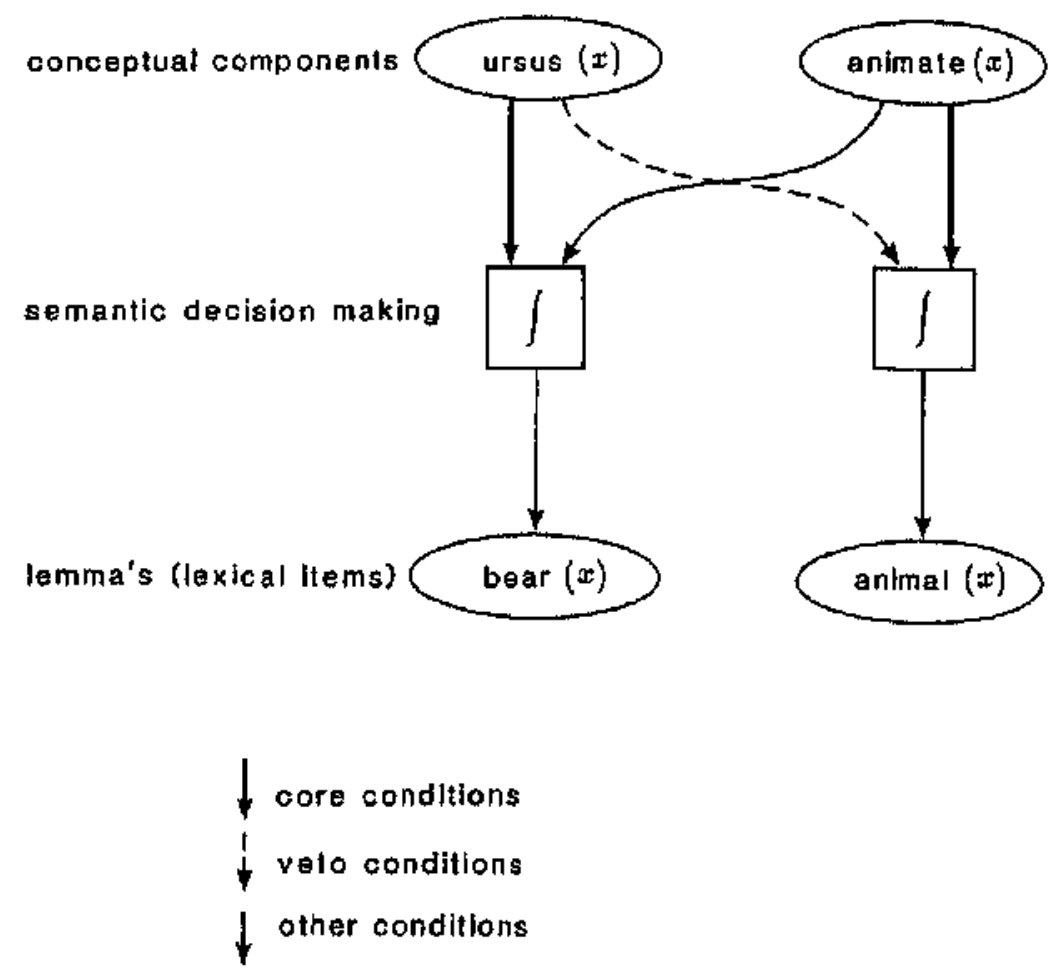

Fig. 2. A fraction of the logical net for lexical selection. The bold arrows depict the effects of core conditions (conditiones sine quibus non), the dotted arrows depict the working of veto conditions.

yields a positive outcome, but also that it attains a certain level of activation. In the present paper, I will not discuss this activation structure any further.

\section{THE TIME COURSE OF LEXICAL ACCESS}

Returning to Figure 1, one can distinguish two views on the time course of lexical access. The first one is the more traditional modular view, which says that there is no phonological encoding before lexical selection and there is, accordingly, no feedback from phonological encoding to lexical selection. The second view is the connectionist picture, which assumes a temporal overlap of lexical selection and phonological encoding, and a continuing interaction between the two processes. These two views are depicted in more detail in Figure 3.

In the classical theories (in particular Garrett's, Kempen's, Butterworth's, Levelt's - see Levelt (1989) for a review) there is an early phase of semantic activation, which rounds up in lexical selection. It is followed by a phase of phonological encoding where only the selected item becomes phonologically encoded. In the connectionist theories (in par- 
THEORIES OF LEXICAL ACCESS
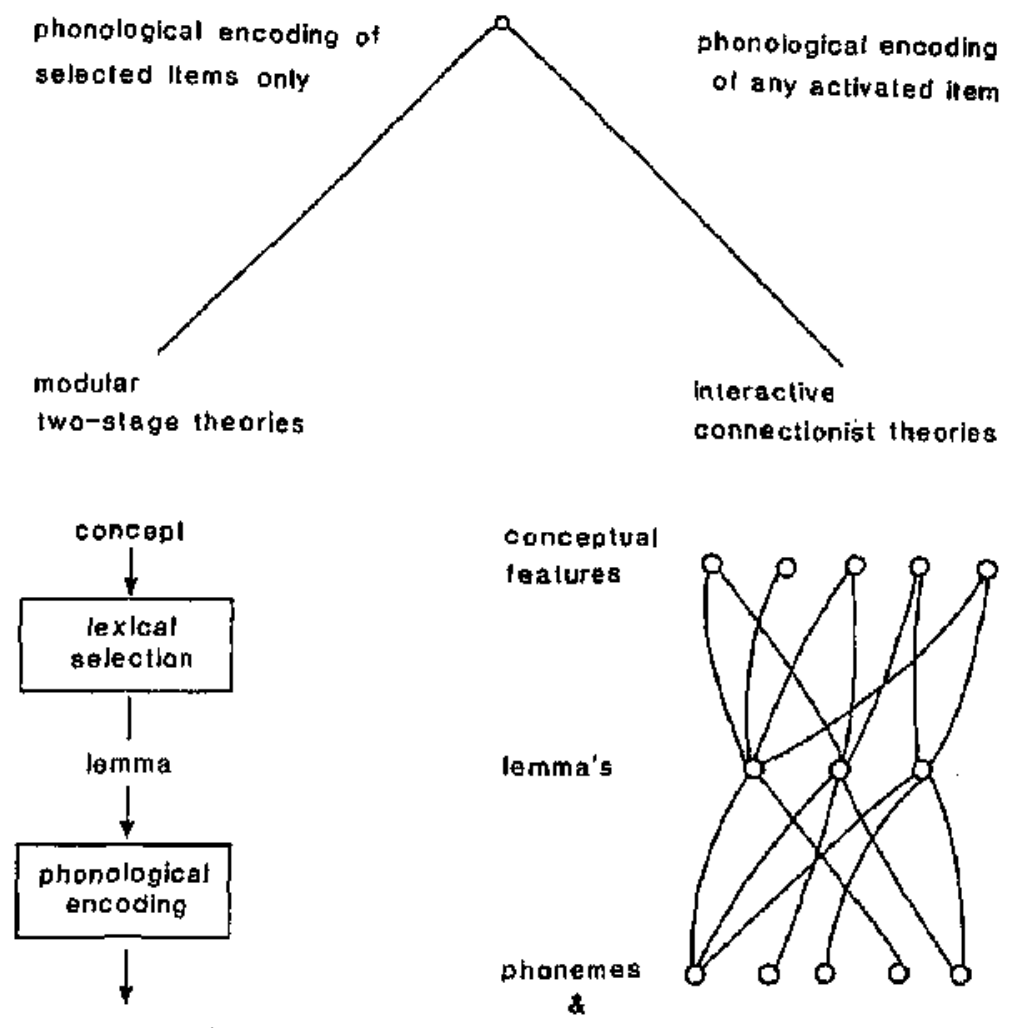

arilculatory plan

phonetic leatures
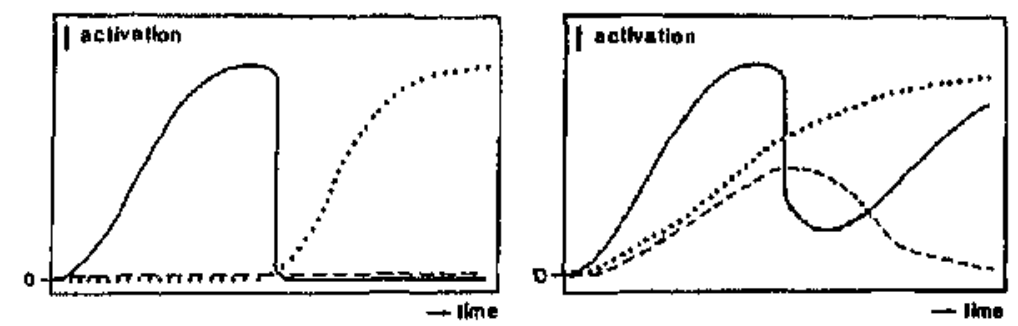

Fig. 3. Two theories of lexical access. The modular two-stage theory (top left) allows for the phonological encoding of the selected target item only. The connectionist theory (top right) predicts phonological activation of semantic alternatives to the target item. The corresponding activation curves are presented at the bottom: Semanic activation of target $(-)$, phonological activation of target $(\ldots$.$) , and phonological activation of semantic$ alternative to target $(-\cdot)$.

ticular Dell's, Stemberger's - see Levelt 1989), not only selected items are phonologically activated, but any semantically activated item.

There are three critical time course predictions proceeding from these theories; they are given at the bottom of Figure 3. The first one concerns 
the course of semantic activation. The modular theory predicts early, no late semantic activation; the connectionist theory (in particular Dell's) predicts early semantic activation and a late rebound of semantic activation, due to feedback from the phonemic to the lemma level. Second, the modular theory predicts late phonological activation, the connectionist theory predicts both early and late phonological activation. Third, the modular theory interdicts phonological activation of semantic alternatives (only the selected item, but no co-activated item becomes phonologically encoded). The connectionist theories, on the other hand, predict phonological activation of semantic alternatives to the target item.

We ${ }^{1}$ performed several experiments to sort these predictions out. The experimental paradigm is presented in Figure 4.

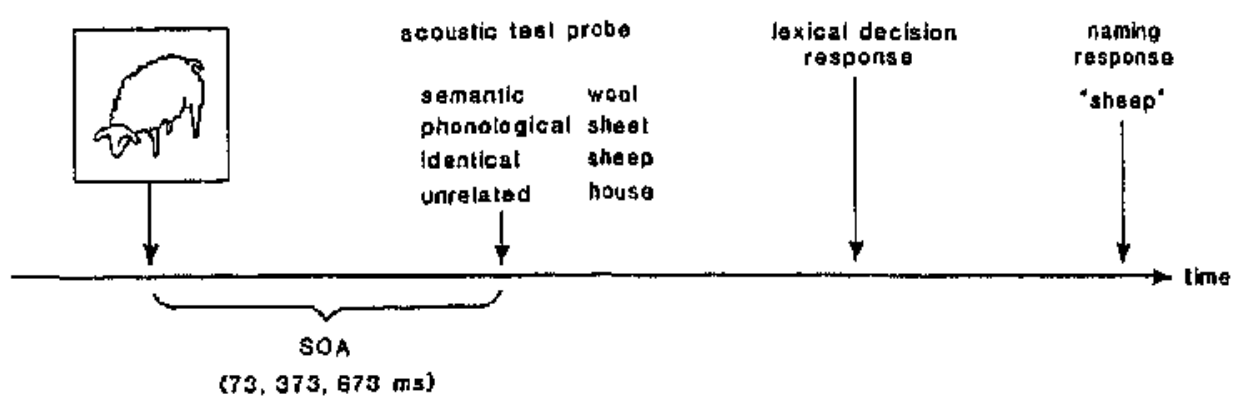

Fig. 4. The experimental procedure of the naming-cum-lexical decision task. See text.

Subjects were asked to perform a picture naming task. A long series of pictures was presented, one by one, and the subject would name each picture as soon as it appeared. Occasionally a secondary so-called "lexical decision" task was given. Shortly after presentation of the picture an acoustic test probe was presented, which could either be a word or a nonword, like sip (word) or sef (non-word). When this happened, the subject was supposed to push a "yes" or a "no" button, correspondingly. This task made it possible to probe into the subject's developing representation in his effort to produce the picture's name. For example, if the picture was one of a sheep the subject would internally generate semantic and phonological representations that were appropriate to the target name sheep. In order to test semantic activation of sheep, we would present as lexical decision probe a word like wool. There is reason to expect that semantic activation of sheep will delay the lexical decision to the acoustic test probe wool. Similarly, we could measure the phonological activation of sheep by presenting a test probe like sheet. In addition, the experiment contained the target word itself as probe (sheep in the example) - which I will not further discuss - and a control condition, namely a test word that is unrelated to the target, for instance house. 
The critical issue is whether a semantic or a phonological test probe shows longer lexical decision latencies than the unrelated test probe. In order to see how the semantic and phonological representations develop over time, we presented the acoustic probe at different delays after the picture. There were three moments: 73,373 , and $673 \mathrm{~ms}$. (on average) after picture onset. These are called "stimulus onset asynchronies", or SOAs.

The lexical decision latencies that we obtained in the experiment $(192$ subjects) are presented in Figure 5 (solid lines). In fact, these data are differences between the measured lexical decision latencies and lexical decision latencies for the same items when presented without concurring naming task. (The positive difference values in the figure show that the concurring naming task generally slowed down the lexical decision response.)

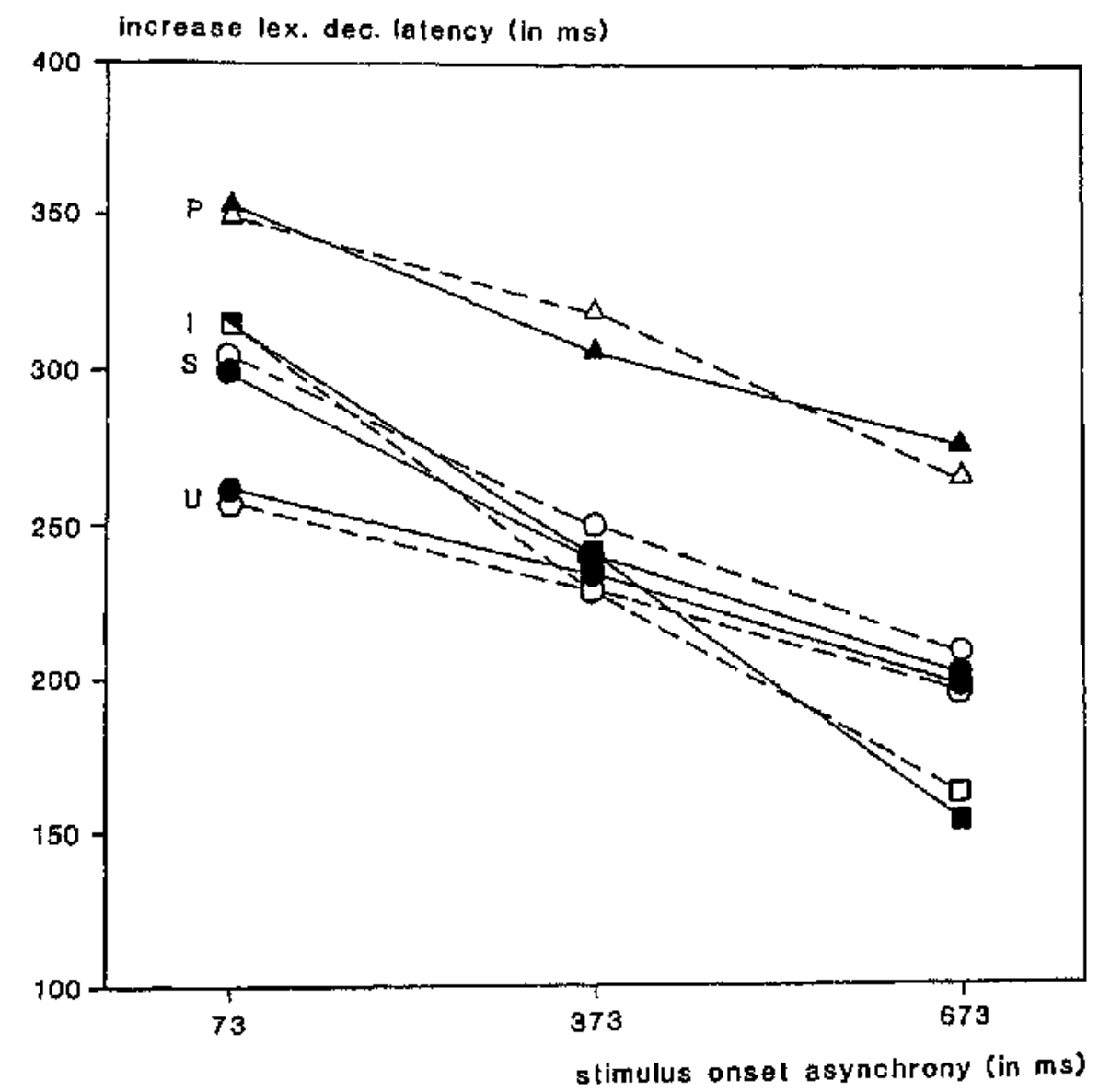

Fig. 5. Increase of lexical decision latencies in the dual naming/lexical decision task (solid lines) for three different types of probe and three SOAs. $\mathrm{S}=$ semantic probes, $\mathrm{P}=$ phonological probes, $\mathrm{I}=$ identical probes, $\mathrm{U}=$ unrelated probes. The totted lines show the fit of the two-stage model to the data. 
As both kinds of model predict, there is good evidence for early semantic activation (at the $73 \mathrm{~ms}$. SOA the latency for the semantic probe is significantly longer than the latency for the unrelated probe). There is, however, no late semantic activation - contrary to the connectionist prediction. As both models predict, there is good evidence for late phonological activation, but seemingly contrary to the modular two-stage model there is evidence for early phonological activation. Hence, these results seem to be equivocal.

I will now argue that the two-stage model should be preferred. The argument is two-way. First, I will show that the two-stage model can give a perfect account of these data. Second, I will report experimental results on the phonological activation of semantic alternatives, which are in support of the two-stage model.

The data in Figure 5 are the statistical result of a huge number of measurements. It is therefore necessary to make a statistical model of this naming-cum-lexical decision task. Figure 6 depicts the model we ${ }^{2}$ developed.

This model incorporates the two-stage modular theory in that there is a strict succession of lexical selection and phonological encoding. The idea is that there will be interference when the semantic stage of naming coincides with the semantic stage of lexical decision, and when the phonological stage of naming coincides with the phonological stage of lexical

NAMING

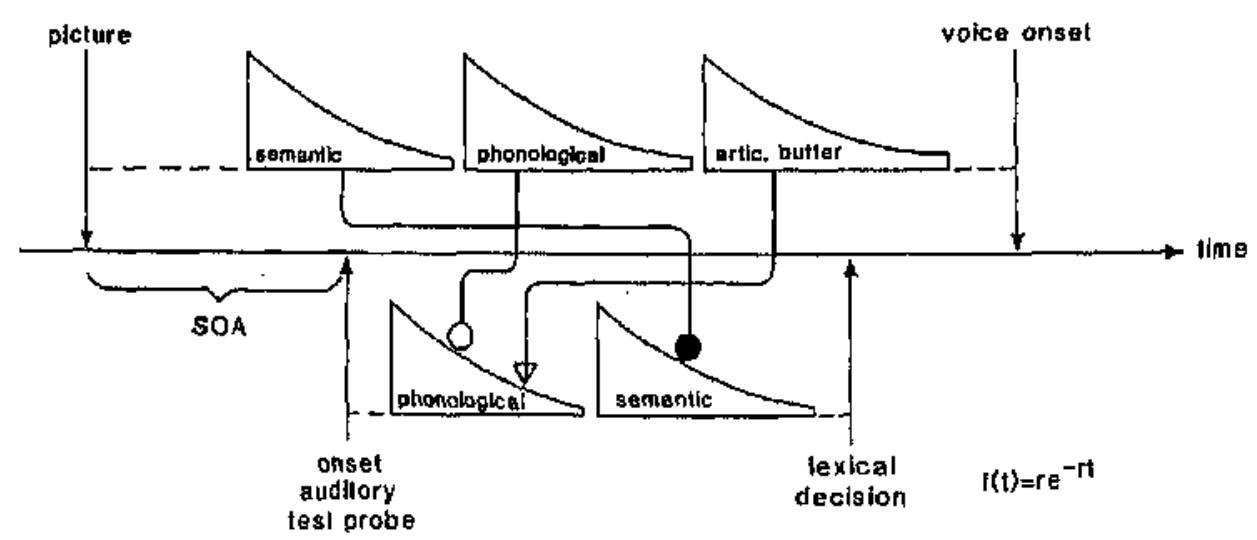

LEXICAL DECISION

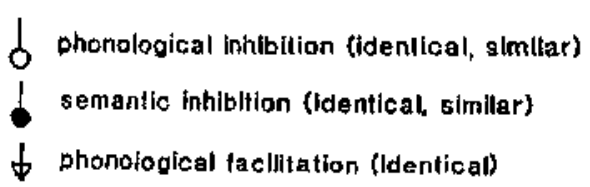

Fig. 6. Mathematical rendering of the two-stage model. 
decision, in case same or similar items are involved in naming and lexical decision. The statistical time distribution of each of these phases is assumed to the exponential, with a characteristic rate parameter for each of the component processes. These rate parameters and the interference parameters can be estimated in order to find a best fit of the model to the data. This we did, and the result is presented in Figure 5, dotted lines. It shows that the data do not contradict the two-stage model. In fact, the fit is statistically perfect.

Turning now to the issue of phonological activation of semantic alternatives, $I$ will report on an experiment that is quite similar to the previous one. But there are two differences. First, we used the short SOA (73 ms.) only, because it gave us both good semantic and good phonological activation in the previous experiment. Second, we used new acoustic test probes. Using again the example where the picture shows a sheep, we used the acoustic probe goat as a semantic probe, and the word goal as a phonological probe. This means that we can test whether the semantic alternative goat is not only semantically, but also phonologically active. In the latter case we should find an effect on goal. And that is what the connectionist theories predict.

Before reporting the results of this experiment, let me first remind you how strong a phonological activation effect we found for target words like sheep in the previous experiment (i.e., the lexical decision latencies for phonological probes like sheet). They are given in Figure 7 , together with the results for the unrelated test probes (such as house) as a comparison.

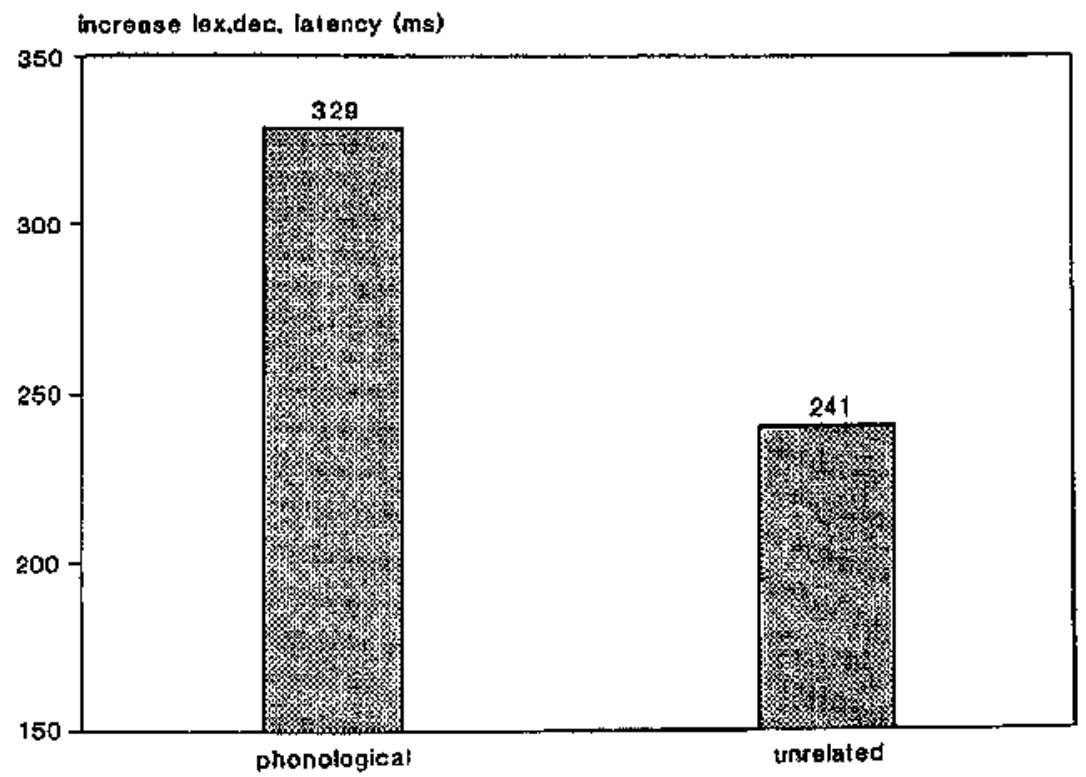

Fig. 7. Increase of lexical decision for probes phonologically related to the target and for unrelated probes. 
Now compare this to the phonological activation we found in the present experiment, i.e., for the semantic alternatives (i.e., for probes such as goal. These results are presented in Figure 8, together with the results for the unrelated test probes (such as house).

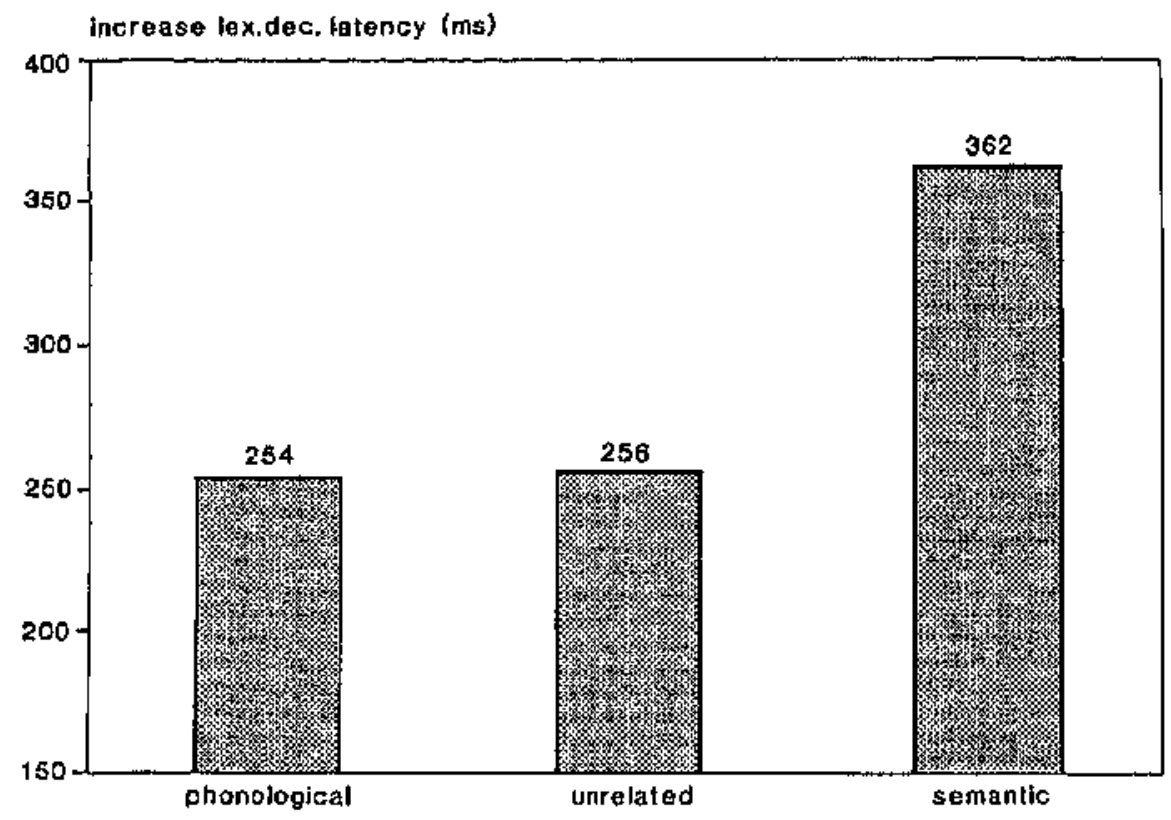

Fig. 8. Increase in lexical decision latency for probes that are phonologically related to a semantic alternative, for unrelated probes, and for probes that are semantic alternatives themselves.

There is not the slightest trace of phonological activation (the result for phonological probes is not different from the result for unrelated probes), contrary to the connectionist predictions. One might, of course object that there was no activation of the semantic alternatives (such as goat to start with. But that is not so. Figure 8 also presents the lexical decision latencies for semantic alternatives such as goat. There is a highly significant effect here if one compares the results for these semantic alternatives to those for unrelated lexical decision probes (such as house).

For a more comprehensive and balanced treatment of the above findings, the reader is kindly referred to Levelt el al. (1991).

\section{CONCLUSION}

Taken together, the reported results support the modular two-stage notion of lexical access. (Further experimental support for this notion can be found in Schriefers et al. 1990.) An important remaining question is: what could be the biological utility of such a modular architecture for lexical access? The obvious answer is that modularity is nature's protection 
against error-proneness of a system. The two components of the lexical accessing mechanism have to perform wildly different tasks. Lexical selection involves fast search in a huge lexicon. Phonological encoding involves the creation of a motor program for a single selected lexical item. If these processes were to interact, one would increase mutual interference without obvious functional advantages. Such interference would lead to errors of lexical selection and of phonological encoding. Though errors of these kinds do occur, their rate is astonishingly low for a process so complex and so fast as lexical access. Errors of lexical selection are probably below one per mille selected items, and errors of phonological encoding are even rarer.

To end with a metaphor: if you want to design a reliable car, you better don't connect the action of the brakes to the action of the steering wheel.

\section{NOTES}

1 Apart from myself, the research team involved Herbert Schriefers, Antje Meyer, and Thomas Pechmann.

2 The model was largely developed by Dirk Vorberg with the assistance of Jaap Havinga.

\section{BIBLIOGRAPHY}

Levelt, Willem J. M.: 1989, Speaking: From Intention to Articulation, MIT Press, Cambridge, Massachusetts.

Levelt, Willem J. M., Herbert Schriefers, Dirk Vorberg, Antje S. Meyer, Thomas Pechmann and Jaap Havinga: 1991, 'The Time Course of Lexical Access in Speech Production. A Study of Picture Naming', Psychological Review 98, 122-142.

Schriefers, Herbert, Antje S. Meyer and Willem J. M. Levelt: 1990, 'Exploring the Time Course of Lexical Access in Production: Picture-word-interference Studies', Journal of Memory and Language 29, 86-102. 